\title{
EFEITO DA PROFUNDIDADE NA EMERGÊNCIA DA PLANTA DANINHA PICÃO PRETO (Bidens pilosa)
}

\author{
EFFECT OF DEPTH IN THE EMERGENCY OF WEED \\ BEGGARTICKS (Bidens pilosa)
}

\author{
Marcelo José Ferreira ${ }^{1}$, Wilson Roberto Cerveira Junior ${ }^{1,2}$, Adilson Ferreira da Silva ${ }^{3}$, Claudinei da Cruz ${ }^{1}$ \\ ${ }^{1}$ Laboratório de Ecotoxicologia e Eficácia dos Agrotóxicos, LEEA, pertencente ao Centro Universitário da \\ Fundação Educacional de Barretos, SP. \\ ${ }^{2}$ Faculdade de Ciências Agrárias e Veterinárias, Unesp, Campus de Jaboticabal, SP. \\ ${ }^{3}$ Citrosuco, Matão, SP.
}

\section{RESUMO}

Para tanto, os objetivos deste estudo foram avaliar a capacidade de emergência da planta daninha (B. pilosa) em diferentes profundidades e determinar sua capacidade de germinação em condição de estufa de demanda biológica de oxigênio (BOD). O experimento foi conduzido em BOD e casa de vegetação, onde foi avaliada a taxa de germinação dos aquênios de $B$. pilosa em diferentes profundidades $(0,0 ; 1,0 ; 2,5 ; 5,0 ; 7,5 ; 10,0 ; 12,5 ; \mathrm{e} 14,0 \mathrm{~cm})$. O delineamento experimental foi inteiramente casualizado (DIC), com oito tratamentos e dez repetições, totalizando 300 sementes por tratamento. Durante o experimento, não ocorreu germinação nas profundidades superiores a $10 \mathrm{~cm}$. Os resultados evidenciaram que a $B$. pilos $a$ na superfície do solo $(0,00 \mathrm{~cm})$ apresenta uma maior taxa de germinação com $79,6 \%$ de seus aquênios e se estende até a profundidade de 7,5 $\mathrm{cm}$ com uma taxa de $16,5 \%$. A partir de $7,5 \mathrm{~cm}$, a $B$. pilosa não apresentou nenhuma germinação durante todo o período experimental. A Bidens pilosa apresentou uma boa capacidade de germinação em menores profundidades, assim, quanto maior a profundidade menor a taxa de emergência.

Palavras-chave: Plantas invasoras, ciclo biológico, manejo, agricultura.

\begin{abstract}
For the purposes of this study, the ability to cause damage to the plant (B. pilosa) at different depths was evaluated and to determine its germination capacity under greenhouse conditions of biological oxygen demand (BOD). The experiment was conducted in BOD and greenhouse where the germination rate of B. pilosa achenes at different depths was evaluated $(0.0 ; 1.0 ; 2.5 ; 5.0 ; 7.5 ; 10.0 ; 12.5$ and $14.0 \mathrm{~cm})$. The experimental design was completely randomized (DIC), with eight treatments and ten repetitions, totaling 300 seeds per treatment. During the experiment there was no germination at depths greater than $10 \mathrm{~cm}$. The results showed that $B$. pilosa on the surface of the soil $(0.00 \mathrm{~cm})$ has a higher germination rate with $79.6 \%$ of its buds and extends to the depth of $7.5 \mathrm{~cm}$ with a rate of $16.5 \%$. After $7.5 \mathrm{~cm}, B$. pilosa did not show any germination during the entire experimental period. However, B. pilosa showed a good germination capacity at lower depths, so it is possible to conclude that, the greater the depth, the lower the emergence rate.
\end{abstract}

Keywords: Weed, biological cycle, management, agriculture.

Autor para Correspondência: MsC. Wilson Roberto Cerveira Junior. Faculdade de Ciências Agrárias e Veterinárias, Unesp, Jaboticabal. E-mail: wilsonrcjunior@gmail.com, Tel. (17)996365405

Recebido para publicação: 19/11/2019

Aceito para publicação: 28/03/2020

https://doi.org/10.4322/1980-0029.142020 


\section{Introdução}

As plantas daninhas com características ruderais podem se estabelecer em ambientes perturbados. Essa é uma das várias características que essas plantas precisaram apresentar para dar continuidade à espécie. As culturas de importância econômica sofrem matocompetição com plantas daninhas, pois essas dificultam várias etapas da produção, aumentando custos e diminuindo produtividade (Christoffoleti \& Passini, 1999). Quando infestam plantações, competem por nutrientes, minerais essenciais, luz, água e espaço. Essas plantas também podem apresentar compostos alelopáticos que podem causar sérios prejuízos ao crescimento, desenvolvimento e produtividade das plantas cultivadas (Kissmann \& Groth, 1999; Ferreira et al., 2007).

As plantas daninhas se destacam pela alta produção de sementes, potencial germinativo elevado, além de grande parte delas possuírem dormência, garantindo sua permanência no solo de três a cinco anos com alta taxa de germinação (Baker, 1974; Lorenzi, 1991). No entanto, essas plantas podem ser de grande importância, uma vez que podem ajudar na proteção do solo e recuperação de áreas degradadas, porém, quando se estabelecem em culturas, passam a ser tratadas como plantas daninhas.

Algumas plantas daninhas têm suas reservas nutritivas limitadas, pois, dependendo da profundidade, elas se tornam plantas dormentes e não conseguem completar seu ciclo de vida, outro fator é a exposição à luz (Klein \& Felippe, 1991). Para a planta daninha conhecida como picão preto (Bidens pilosa), a presença de luz apresenta efeito na emergência de suas sementes, além da necessidade de uma determinada variação térmica e disponibilidade de água e oxigênio para sua emergência (Adegas et al., 2003).

Dentre as plantas daninhas, o picão preto (Bidens pilosa) tem chamado a atenção de produtores e pesquisadores por se tratar de uma planta de difícil controle. Apresenta ciclo curto, grande produção de sementes (aquênios), adaptação do propágulo reprodutivo, com estruturas especializadas, que permite maior leveza e aderência a animais e tecidos, além da grande deposição de fitomelanina que protege o pericarpo, diminuindo injúrias e/ou predação. Uma alta densidade tricomática, baixa densidade estomática e alto teor de cera epicuticular principalmente na face adaxial, como barreiras à entrada de herbicidas (Santos, 2011).

A B. pilosa apresenta fotoblastismo positivo, portanto, a luz estimula a germinação, aumentando de $50 \%$ no escuro para $70 \%$ em presença de luz, isso mantendo uma temperatura constante durante todo o experimento (Klein \& Felippe, 1991). De acordo com Kissmann \& Groth (1999), uma planta de B. pilosa é capaz de gerar até 3000 sementes; muitas destas sementes podem permanecer dormentes por vários anos, só esperando uma condição favorável para sua emergência.

Assim, o conhecimento sobre a profundidade de germinação da $B$. pilosa, que levam a medidas simples como o revolvimento do solo, pode tornar muito eficiente e mais barato o controle, dependendo da planta que se encontra no local. Para tanto, os objetivos deste estudo foram avaliar a capacidade de emergência da planta daninha (B. pilosa) em profundidades e determinar sua capacidade de germinação em condição de estufa de demanda biológica de oxigênio (BOD).

\section{Material e Métodos}

As sementes utilizadas na condução deste experimento foram coletadas a partir de plantas cultivadas em casa de vegetação que apresentavam bom estado sanitário e nutricional. Após coletadas, foram armazenadas em bandejas de alumínio para redução do teor de umidade.

Para avaliação da capacidade de germinação das sementes de $B$. pilosa, foram utilizadas caixas plásticas tipo gerbox contendo 3 papéis para germinação do tipo germotest. Foram utilizadas 15 caixas gerbox com 10 sementes em cada caixa, totalizando 150 sementes.

Após a semeadura, foram colocados $8,5 \mathrm{ml} \mathrm{de}$ água e as unidades experimentais foram transferidas para uma estufa de demanda biológica de oxigênio (BOD) com fotoperíodo de 12 horas de luz e temperatura de $25^{\circ} \mathrm{C}$. As avaliações da germinação foram realizadas em 7,14 e 21 dias após a semeadura. Os dados obtidos foram utilizados no cálculo do potencial de inibição da emergência da planta teste e foram submetidos à análise de variância (ANOVA) e suas médias comparadas pelo teste de Tukey a $5 \%$ de probabilidade.

Para a condução do experimento em casa de vegetação, o solo para montagem dos vasos foi coletado no campus do Centro Universitário da Fundação educacional de Barretos com 
coordenadas geográficas aproximadas de Latitude Sul: $20^{\circ} 33^{\prime} 26^{\prime \prime}$ e Longitude Oeste: 48³4'04”, com altitude de 530 metros, na camada arável até $15 \mathrm{~cm}$ de profundidade. A seguir, foi peneirado em peneira de malha $5 \mathrm{~mm}$ para obter um solo com um padrão granulométrico e livre de impurezas indeterminadas ao experimento.

Para a quantidade de água a ser utilizada no experimento, foi realizada a determinação da capacidade de saturação de água no solo. Após este procedimento, o solo foi disposto em vasos de plástico com capacidade para 5 litros até a borda e completado de água até atingir o ponto de saturação. Feitos os testes de saturação, chegouse à conclusão de que o turno de irrigação seria de $150 \mathrm{ml}$.

Cada vaso teve o fundo revestido com uma folha de papel filtro para que não houvesse a perda de solo. A semeadura da B. pilosa foi realizada utilizando uma régua (gabarito de profundidade) que era colocada dentro do vaso para que se tivesse uma marcação mais precisa da profundidade. Dando sequência, completava-se com solo até a profundidade desejada. Assim, foram colocadas as sementes de $B$. pilosa e posteriormente foi acrescentado solo até atingir a borda do vaso. Os tratamentos foram 0,$0 ; 1,0 ; 2,5 ; 5,0 ; 7,5 ; 10,0 ; 12,5 ; \mathrm{e} 14,0 \mathrm{~cm}$ de profundidade de semeadura. Cada vaso foi considerado uma unidade experimental e foram colocadas 30 sementes por vaso. O delineamento experimental adotado foi inteiramente casualizado (DIC), com oito tratamentos e dez repetições, em condição de casa de vegetação.
As avaliações de germinação foram realizadas em $0,1,3,5,7,9,11,15,21,25,30,35,40,45$ e 60 dias após a semeadura (DAS). Nas avaliações, foram realizados a contagem das plantas emergidas, o cálculo de porcentagem de emergência e desvio padrão.

\section{Resultados e discussão}

$\mathrm{Na}$ avaliação da germinação de sementes de B. pilosa em condição de BOD, observou-se maior porcentagem de germinação no primeiro dia, com $32 \%$. Nas demais avaliações, a porcentagem de germinação decaiu (Tabela 1).

Em casa de vegetação, observou-se que as sementes de $B$. pilosa iniciaram sua emergência no terceiro dia após a semeadura, nas profundidades de 0,0 até $7,5 \mathrm{~cm}$, similar ao descrito para Brachiaria decumbens com germinação entre 0,0 e $7,0 \mathrm{~cm}$ de profundidade (Silva et al., 2014). Aos três dias após a semeadura, na profundidade de $1,0 \mathrm{~cm}$, verificouse maior taxa de emergência, quando comparada às demais profundidades (Tabela 2 ). No quinto $\mathrm{e}$ no sétimo dia após a semeadura, a profundidade que apresentou maior taxa de germinação foi a de 2,5 cm com 3,9 e 6,2 plantas, respectivamente (Tabela 2). Aos nove dias após a semeadura, nas profundidades de 1,0 e $5,0 \mathrm{~cm}$, ocorreu emergência de 3,2 plantas. Nas avaliações de 11 e 15 dias após semeadura, o número de plantas germinadas decaiu em todas as profundidades avaliadas (Tabela 2). No entanto, na profundidade de $0,00 \mathrm{~cm}$, a taxa de germinação foi constante até o término da avaliação (Tabela 2).

Tabela 1. Número médio de sementes de Bidens pilosa germinadas e porcentagem de germinação em condições de estufa de demanda biológica de oxigênio (BOD).

\begin{tabular}{clllllll}
\hline Avaliação (dia) & $\mathbf{1}$ & $\mathbf{3}$ & $\mathbf{5}$ & $\mathbf{7}$ & $\mathbf{9}$ & $\mathbf{1 1}$ & $\mathbf{1 4}$ \\
\hline Número de sementes & $48,0 \mathrm{a}$ & $9,0 \mathrm{~b}$ & $1,0 \mathrm{c}$ & $4,0 \mathrm{c}$ & $2,0 \mathrm{c}$ & $6,0 \mathrm{~b}$ & $8,0 \mathrm{~b}$ \\
$\mathbf{\%}$ & 32,0 & 6,0 & 0,7 & 2,6 & 1,3 & 4,0 & 5,3 \\
\hline
\end{tabular}

Médias seguidas de mesma letra na coluna não diferem estatisticamente entre si pelo teste Tukey $(\mathrm{p}>0,05)$.

Tabela 2. Média e desvio padrão de plantas de B. pilosa emergidas durante o período experimental em função da profundidade $(\mathrm{cm})$.

\begin{tabular}{ccccccccccccc}
\hline $\begin{array}{c}\text { Prof. } \\
\text { (cm) }\end{array}$ & $\mathbf{1 0}$ & $\mathbf{1 0}$ & $\mathbf{7}$ & $\mathbf{9}$ & $\mathbf{1 1}$ & $\mathbf{1 5}$ & $\mathbf{2 1}$ & $\mathbf{2 5}$ & $\mathbf{3 0}$ & $\mathbf{3 5}$ & $\mathbf{4 0}$ & $\mathbf{4 5}$ \\
\hline $\mathbf{0 , 0 0}$ & $5,2 \pm 2,0$ & $3,2 \pm 1,5$ & $5,8 \pm 3,0$ & $3,2 \pm 3,1$ & $3,2 \pm 2,1$ & $0,3 \pm 0,4$ & $0,6 \pm 1,5$ & $0,6 \pm 0,7$ & $0,6 \pm 0,8$ & $0,4 \pm 0,7$ & $0,4 \pm 1,2$ & $0,4 \pm 1,2$ \\
$\mathbf{1 , 0 0}$ & $6,7 \pm 3,8$ & $3,1 \pm 2,6$ & $3,3 \pm 1,8$ & $0,9 \pm 0,8$ & $0,8 \pm 1,2$ & $0,1 \pm 0,3$ & $0,8 \pm 1,4$ & - & - & $0,10 \pm 0,3$ & $0,1 \pm 0,3$ & - \\
$\mathbf{2 , 5 0}$ & $3,7 \pm 1,6$ & $3,9 \pm 1,4$ & $6,2 \pm 2,7$ & $2,1 \pm 1,3$ & $1,2 \pm 0,1$ & $0,7 \pm 1,2$ & - & - & - & - & - & - \\
$\mathbf{5 , 0 0}$ & $0,8 \pm 0,6$ & $1,7 \pm 1,4$ & $5,6 \pm 2,7$ & $3,2 \pm 1,6$ & $1,8 \pm 2,2$ & $0,5 \pm 0,8$ & $0,1 \pm 0,3$ & $0,1 \pm 0,3$ & - & - & - & - \\
$\mathbf{7 , 5 0}$ & $0,1 \pm 0,3$ & $0,1 \pm 0,3$ & $1,8 \pm 2,6$ & $1,5 \pm 2,0$ & $0,3 \pm 0,4$ & $0,1 \pm 0,3$ & $0,4 \pm 0,7$ & - & $0,2 \pm 0,4$ & - & - & - \\
\hline
\end{tabular}

(-) indica ausência de emergência no período de avaliação. 
Tabela 3. Número total e porcentagem de emergência de sementes de B. pilosa em diferentes profundidades (cm).

\begin{tabular}{lrrrrrrcc}
\hline PROFUNDIDADE (CM) & $\mathbf{0 , 0}$ & $\mathbf{1 , 0}$ & $\mathbf{2 , 5}$ & $\mathbf{5 , 0}$ & $\mathbf{7 , 5}$ & $\mathbf{1 0 , 0}$ & $\mathbf{1 2 , 5}$ & $\mathbf{1 4 , 0}$ \\
No TOTAL DE EMERGÊNCIA & 239,0 & 175,1 & 185,8 & 151 & 49,5 & - & - & - \\
\% DE EMERGÊNCIA & 79,6 & 58,3 & 61,9 & 50,3 & 16,5 & - & - & - \\
\hline
\end{tabular}

(-) indica ausência de emergência no período de avaliação.

De acordo com o trabalho realizado por Oliveira \& Norsworthy (2006), o aumento da profundidade das sementes de Ipomoea lacunosa diminui a taxa de germinação, similar ao observado no presente estudo para $B$. pilosa. No entanto, ao atingir a profundidade de $4,0 \mathrm{~cm}$, I. lacunosa apresentou taxa de germinação de $50 \%$, similar ao observado para B. pilosa na profundidade de $5,0 \mathrm{~cm}$ (Tabela 3 ).

Nas maiores profundidades testadas $(10,0 ; 12,5$; e 14,0 cm), não ocorreu emergência da planta daninha em nenhum dos períodos de avaliação. Portanto os resultados mostraram que a profundidade dos aquênios interferiu na emergência das plântulas de $B$. pilosa e ocorreu limitação na germinação em função da profundidade.

No total de sementes, em 300 sementes por tratamento, ocorreu emergência de 239 plantas em 0,0 , que foi a profundidade que mais emergiu, enquanto em 7,5 cm emergiram 49 plantas (Tabela 3). A emergência neste estudo diferiu do obtido para Ipomoea asarifolia, que apresentou maior taxa de germinação em profundidade superior a $10 \mathrm{~cm}$ devido ao ressecamento das sementes (Dias Filho, 1996), porém foi similar à da $B$. decumbens com maior taxa de germinação entre 1,0 e $7,0 \mathrm{~cm}$ de profundidade (Silva et al., 2014).

Com base nos estudos realizados por Sahoo \& Jha (1998) e Muniz Filho et al. (2004), B. pilosa apresentou germinação satisfatória até a profundidade de $2,0 \mathrm{~cm}$, diferindo do ocorrido neste presente estudo em que a melhor profundidade foi de $0,0 \mathrm{~cm}$, onde ocorreu uma taxa de 79,6\% de emergência das sementes. Para Sharma (1992), os aquênios de erva-de-touro (Tridax procumbens) têm um maior número de emergência na superfície até a profundidade de $5 \mathrm{~cm}$ de profundidade, enquanto, para Toledo et al. (1993), a Xanthium strumarium apresentou germinação em profundidades superiores a $15 \mathrm{~cm}$, o que foi o dobro da profundidade máxima atingida pela $B$. pilosa obtida neste estudo.

Orzari (2013) verificou que I. grandifolia apresentou uma taxa de germinação na superfície de $36 \%$; nas profundidades de 0,5 a $5 \mathrm{~cm}$, oscilou de $29,39 \%$ a $16,37 \%$; e chegando a $2,32 \%$ em $20 \mathrm{~cm}$. Para Ipomoea nil, ocorreu a mesma sequência de resultados com taxa de $25,48 \%$ na superfície e de $2,28 \%$ em $20 \mathrm{~cm}$, diferindo do observado para B. pilosa com maior taxa de germinação entre a superfície e $7,5 \mathrm{~cm}$ e sem germinação a partir de $10 \mathrm{~cm}$ de profundidade. Orzari (2013) também destacou que Merremia aegyptia apresentou alta taxa de germinação superficial $(61,67 \%)$ similar à taxa de germinação da $B$. pilosa, contudo ela demonstrou que tem alto vigor de germinação na profundidade de $10,0 \mathrm{~cm}$ com uma taxa de $30,62 \%$ e chegando aos $20 \mathrm{~cm}$ de profundidade com $15,21 \%$ de emergência. Estes resultados também podem ser úteis para explicar as grandes densidades dessa planta daninha em áreas sob plantio direto, onde as sementes são depositadas nas superfícies do solo.

\section{Conclusões}

O picão preto (B. pilosa) apresentou uma boa capacidade de germinação em menores profundidades e menor capacidade de germinação com o aumento da profundidade. As características da biologia reprodutiva de $B$. pilosa é importante para a formação de densos bancos de sementes em solos submetidos ao preparo convencional, onde grande parte das sementes é incorporada.

\section{Referências}

Adegas, F. S., Voll, E., \& Prete, C. E. C. (2003). Embebição e germinação de sementes de picão-preto (Bidens pilosa). Planta Daninha, 21(1), 21-25.

Baker, H. G. (1974). The evolution of weeds. Annual Review of Ecology and Systematics, 5(1), 1-24.

Christoffoleti, P. J., \& Passini, T. (1999). Manejo integrado de plantas daninhas na cultura do feijão. In A. L. Fancelli \& D. Dourado Neto. Feijão irrigado: estratégias básicas de manejo (pp. 8097). Piracicaba: LPV/ESALQ/USP.

Dias Filho, M. B. (1996). Germination and emergence of Stachytarpheta cayennensis and Ipomoea asarifolia. Planta Daninha, 14(2), 118-126. 
Ferreira, M. C., Souza, J. R. P., \& Faria, T. J. (2007). Potenciação alelopática de extratos vegetais na germinação e no crescimento inicial de picão preto e alface. Ciência e Agrotecnologia, 31(4), 1054-1060.

Kissmann, K. G., \& Groth, D. (1999). Plantas infestantes e nocivas (2. ed., Tomo II, 978 p.). São Paulo: BASF.

Klein, A. L., \& Felippe, G. M. (1991). Efeito da luz na germinação de sementes de ervas invasoras. Pesquisa Agropecuária Brasileira, 26(7), 955-966.

Lorenzi, H. (1991). Plantas daninhas do Brasil: terrestres, aquáticas, parasitas, tóxicas e medicinais (2. ed., 440 p.). Nova Odessa: Plantarum.

Muniz Filho, A., Carneiro, P. T., Cavalcanti, M. L. F., Albuquerque, R. C. (2004). Capacidade de emergência de picãopreto em diferentes profundidades de semeadura. Revista de Biologia e Ciências da Terra, 4(1), 1-6.

Oliveira, M., \& Norsworthy, J. K. (2006). Pitted morningglory (Ipomoea lacunosa) germination and emergence as affected by environmental factors and seeding depth. Weed Science, 54(5), 910-916.
Orzari, I. (2013). Germinação de espécies da família Convolvulaceae sob diferentes condições de luz, temperatura e profundidade de semeadura. Planta Daninha, 31(1)

Sahoo, U. K., \& Jha, L. K. (1998). Effect of depth and duration of burial on seed viability and dormancy of Bidens pilosa L. and Richardsonia pilosa H. B. K. Seed Research, 25(1), 5-10.

Santos, J. B. (2011). Picão-preto: uma planta daninha especial em solos tropicais. Planta Daninha, 29(n. spe), 1159-1172.

Sharma, B. M. (1992). Preliminary ecological studies on lithophytes and chasmophytes in South-West Nigeria. Feddes Repertorium, 103(3-4), 235-242.

Silva, A. F., Pitelli, R. A., Cruz, C., Garlich, N., Florêncio, T., \& Yamauchi, A. K. F. (2014). Emergency Brachiaria decumbens a function of depth of seed in soil profile. Nucleus Animalium, 6(2), 29-36.

Toledo, R. E. B., Kuva, M. A., \& Alves, P. L. C. A. (1993). Fatores que afetam a germinação e a emergência de Xanthium strumarium L.: dormência, qualidade da luz e profundidade de semeadura. Planta Daninha, 11(1-2), 1520. 